\title{
CARACTERIZAÇÃO DE PLACAS DE MEMÓRIA DE COMPUTADORES OBSOLETOS VISANDO A RECUPERAÇAO DE PRATA POR MEIO DE NANOPARTÍCULAS*
}

\author{
Marcos Paulo Kohler Caldas ${ }^{1}$ \\ Mariana Alves de Carvalho ${ }^{2}$ \\ Viviane Tavares de Moraes ${ }^{3}$ \\ Jorge Alberto Soares Tenório ${ }^{4}$ \\ Denise Crocce Romano Espinosa ${ }^{5}$
}

\section{Resumo}

A combinação do elevado volume gerado de resíduos de equipamentos eletroeletrônicos (REEE) com o aumento da obsolescência programada faz com que haja a necessidade de se pensar em alternativas para a disposição destes resíduos sem impacto ao meio ambiente e à saúde humana, como a reciclagem com posterior recuperação de metais. Para isso, é necessário conhecer a natureza dos materiais constituintes do resíduo. A Placa de Circuito Impresso (PCl) é um importante representante dos REEE, pois está presente em grande parte dos equipamentos eletrônicos, como nos computadores. Sua composição heterogênea possui um alto teor de metais, alguns deles preciosos, que representam quase a totalidade do valor intrínseco da placa, justificando a importância da reciclagem para recuperação dos mesmos. No presente trabalho foi realizada a caracterização de uma placa de memória de computadores obsoletos para a posterior recuperação dos metais preciosos nela contidos na forma de nanopartículas. Conclui-se que a placa de memória possui 18,9\% de metais, $48,4 \%$ de cerâmicas e $32,7 \%$ de polímeros em massa. Dentre os metais o Cu é o que aparece de forma mais expressiva, constituindo 13,83\% em massa da placa. Os metais preciosos $\mathrm{Ag}$ e $\mathrm{Au}$ constituem 0,053\% e 0,069\% em massa, respectivamente, porém seu alto valor de mercado justifica a viabilidade de sua recuperação.

Palavras-chave: Reciclagem; Caracterização; Nanopartículas; Metais preciosos.

\section{CHARACTERIZATION OF MEMORY BOARDS OF OBSOLETE COMPUTERS FOR THE RECOVERY OF SILVER BY MEANS OF NANOPARTICLES}

\section{Abstract}

Combination of high generated amount of waste electrical and electronic equipments (WEEE) with the increase of programmed obsolescence demands the need of thinking in alternatives for the disposal of these residues without environmental and human health impact. To achieve this, it is necessary to know the nature of constituents material of the residue. Printed circuit board (PCB) is an important representant of WEEE, because it's present in almost every electronic equipments, including computers. Its heterogen composition has a high content of metals, some of them precious, which represents almost all the intrinsic value of the board, what justify the importance of recycling to recover them. On this work, it was made the characterization of a memory board from obsolete computers to further recovery of precious metal in form of nanoparticles. It was concluded that the board has a weight percentage of $18.9 \%$ of metals, $48.4 \%$ of ceramics and $32,7 \%$ of polymers. Among the metals, $\mathrm{Cu}$ appears in a most expressive way, representing a weight percentage of $13.83 \%$. Precious metals $\mathrm{Ag}$ and $\mathrm{Au}$ represent $0.053 \%$ and $0.069 \%$ of the board, respectively, but their high market value justify the recovery viability.

Keywords: Recycling; Characterization; Nanoparticles; Precious metals.

Doutor em Eng. Química, Depto. de Engenharia Química, Escola Politécnica, USP, São Paulo, SP, Brasil. Mestranda em Eng. Química, Depto. de Eng. Química, Escola Politécnica, USP, São Paulo, SP, Brasil. Doutora em Engenharia Metalúrgica e de Materiais, Professora do SESI - São Bernardo do Campo, São Bernardo do Campo, SP, Brasil.

4 Professor Titular, Departamento de Engenharia Química, Escola Politécnica, USP, São Paulo, SP, Brasil.

5 Professora Associada, Departamento de Engenharia Química, Escola Politécnica, USP, São Paulo, SP, Brasil. 


\section{INTRODUÇÃO}

A produção de eletroeletrônicos cresce exponencialmente à medida que suas aplicações se diferenciam em todos setores da sociedade (1). Por outro lado, o ciclo de vida destes produtos tem diminuído em decorrência da obsolescência causada pelo avanço tecnológico $(2,3)$. Isso leva a um aumento alarmante da geração de resíduos eletroeletrônicos, os chamados REEE, que demandam que haja uma estratégia para que sua disposição final não cause danos ao ambiente e ao bemestar humano (4). Diretrizes propostas pelo Parlamento Europeu acerca da gestão de resíduos eletroeletrônicos (5) e do uso de substâncias perigosas na fabricação de eletroeletrônicos (6) tiveram o intuito de contribuir no sentido de chamar atenção da sociedade para este aspecto, com propostas para mitigar os efeitos negativos de seu processo produtivo e disposição final (7).

Dentre os REEE, as Placas de Circuito Impresso (PCIs) se destacam por estarem presentes na maior parte dos equipamentos eletrônicos, de forma que representam um grande volume dos resíduos no final do ciclo de vida (4). A composição das PCls é complexa devido à origem heterogênea de seus materiais constituintes. São materiais cerâmicos, poliméricos e metálicos, em proporções variadas. A fração metálica, que varia em torno de $30 \%$, abrange uma ampla gama de metais, como $\mathrm{Cu}, \mathrm{Fe}, \mathrm{Al}, \mathrm{Ni}, \mathrm{Zn}, \mathrm{Sn}$, metais preciosos como Al, Au e Pd, e metais potencialmente tóxicos como $\mathrm{Hg}$, Cd e $\mathrm{Pb}(1,8)$.

Se dispostos no meio ambiente de forma indiscriminada, os componentes das PCls entram em contato com o solo, contaminando os ecossistemas locais e podendo ser transportados por corpos d'água, atingindo e causando problemas à saúde humana. Considerando o elevado volume gerado, isso pode se dar em larga escala (9). Por isso, a reciclagem de REEE torna-se uma alternativa interessante para o destino de PCls e outros REEE no fim do ciclo de vida.

Além do viés ambiental, a reciclagem das PCls é atrativa economicamente, dada a possibilidade de recuperação dos metais em sua composição. Tal recuperação acaba por preservar os recursos naturais, pois a aplicação de metais de reciclagem reduz a demanda por metais virgens oriundos de minérios (8).

Metais preciosos como Ag e Au são utilizados no processo de fabricação de PCls devido às suas características que os tornam materiais bastante estáveis e com propriedades condutoras de elevado desempenho (10). Além disso, possuem um alto percentual do valor intrínseco do resíduo, ainda que estejam presentes em proporções muito menores que os outros metais, com teores menores que $1 \%$. Isso torna o resíduo uma fonte secundária promissora em detrimento dos minérios destes metais $(1,7)$.

A reciclagem das PCls pode ser feita por diversos processos de acordo com o objetivo e características do resíduo. Para a recuperação de metais, pode-se utilizar processos hidrometalúrgicos (11), pirometalúrgicos (3) e biohidrometalúrgicos (1). Todos os processos possuem suas vantagens e limitações, e o conhecimento da composição do resíduo dará respaldo para a escolha daquele que aparece como a melhor opção.

O presente trabalho objetiva caracterizar uma $\mathrm{PCl}$, a placa de memória de computadores obsoletos, visando a recuperação de metais preciosos por meio de rotas hidrometalúrgicas posteriores. 


\section{MATERIAIS E MÉTODOS}

As PCls utilizadas foram cedidas pelo Centro de Descarte e Reuso de Resíduos de Informática da Universidade de São Paulo (CEDIR/USP), conforme Figura 1. Foram selecionadas placas lead free devido a substituição do chumbo por compostos de prata. Peças removíveis de maior tamanho como dissipadores de alumínio e componentes de refrigeração foram retirados manualmente.

$\mathrm{Na}$ sequencia as PCls foram: cominuidas; caracterizadas por digestão em água régia, ICP-OES e perda ao fogo.

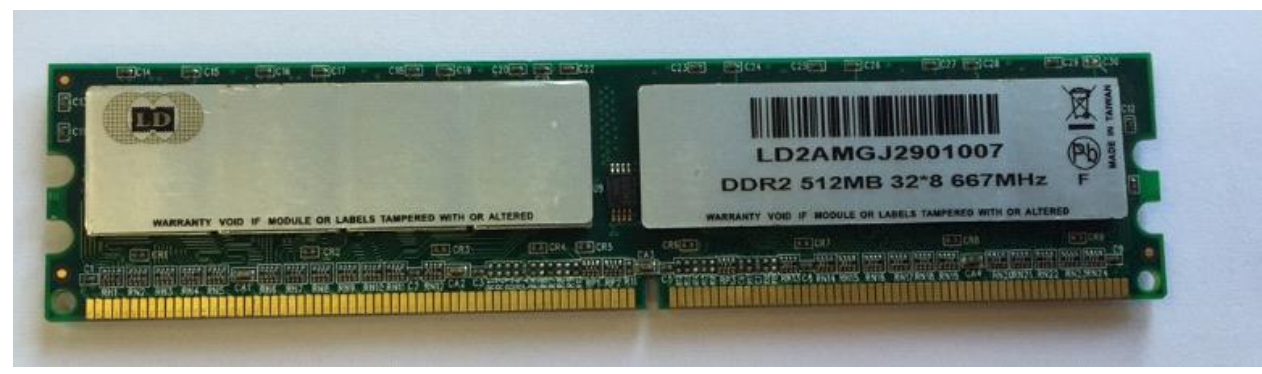

Figura 1. Placa de memória lead free utilizada na caracterização.

\subsection{Cominuição}

Para redução do tamanho das PCls e facilitar sua moagem utilizou-se uma guilhotina manual para cortar as placas em duas partes. Em seguida as PCls foram cominuidas em duas etapas, inicialmente passaram por um moinho de facas modelo RONE FA2305 com grelha de $9 \mathrm{~mm}$ e depois por um moinho de martelos modelo MDM 18/18 da marca Astecma com grelha de $2 \mathrm{~mm}$. Após as etapas de cominuição das PCls, o material moído foi quarteado visando melhor homogeneização das amostras.

\subsection{Caracterização}

\subsubsection{Digestão em água régia}

Uma fração quarteada de $5 \mathrm{~g}$ foi solubilizada pelo contato direto com uma solução de água régia na relação de 3:1 $\left(\mathrm{HCl}: \mathrm{HNO}_{3}\right)$ na proporção sólido líquido de 1:20 [13] ( $1 \mathrm{~g}$ da amostra para $20 \mathrm{ml}$ de solução de água régia), portanto $5 \mathrm{~g}$ da amostra foi colocada em contato direto com $100 \mathrm{ml}$ de solução ácida.

A solubilização dos metais em água régia foi feita durante 24 horas a temperatura ambiente.

Após esse período o solido não lixiviado foi filtrado do licor lixiviado por meio de filtração simples utilizando-se papel de filtro quantitativo de porosidade $0,75 \mu \mathrm{m}$. $\mathrm{O}$ resíduo não lixiviado foi lavado com água deionizada e mantido em estufa a $60^{\circ} \mathrm{C}+/-$ $5^{\circ} \mathrm{C}$ por $24 \mathrm{~h}$ para secar. Uma alíquota de $10 \mathrm{ml}$ foi coletada do licor lixiviado para análise química em ICP-OES e determinação da quantidade de metais. 


\subsubsection{Espectrometria de emissão óptica com plasma acoplado indutivamente (ICP-OES)}

Para a determinação da concentração de metais presentes no licor de lixiviação foi realizada análise de ICP-OES.

\subsubsection{Perda ao fogo}

O ensaio de perda ao fogo foi realizado em forno tipo mufla a $800^{\circ} \mathrm{C}$ por uma hora. Uma fração de aproximadamente $2 \mathrm{~g}$ foi colocada em navícula de porcelana previamente pesada. A navícula foi inserida no forno, aquecido a $800^{\circ} \mathrm{C} \mathrm{com} \mathrm{taxa} \mathrm{de}$ aquecimento de $10^{\circ} \mathrm{C} /$ minuto, e permaneceu nessa temperatura por uma hora. Após esse tempo o forno foi desligado resfriando-se até a temperatura ambiente.

O material não volatizado presente na navícula corresponde ao material cerâmico presente na fração estudada. A diferença entre a massa inicial a e a massa calcinada corresponde aos polímeros presentes na $\mathrm{PCl}$.

\section{RESULTADOS E DISCUSSÃO}

\subsection{Cominuição}

Após a cominuição no moinho de facas o material moído foi pesado e apresentou perda de $0,17 \%$. No moinho de martelos a perda foi de $1,29 \%$, resultando em uma perda total de material durante a cominuição de 1,46\%. A perda foi calculada pela diferença entre a massa inicial da amostra e a massa final após a respectiva cominuição, o que indica a baixa perda de materiais, principalmente de finos.

\subsection{Caracterização}

\subsubsection{Digestão em água régia}

Através do ensaio de digestão em água régia pôde-se determinar a quantidade de metais que compõe a $\mathrm{PCl}$ através do cálculo de perda de massa da amostra lixiviada. A Figura 2 apresenta a quantidade de metais presentes na placa de circuito impresso como porcentagem de material solúvel. Pode ser observado que a placa é composta de $19 \%$ de metais, isto é, material solúvel e $81 \%$ de materiais polímeros e cerâmicos, isto é, material insolúvel. 


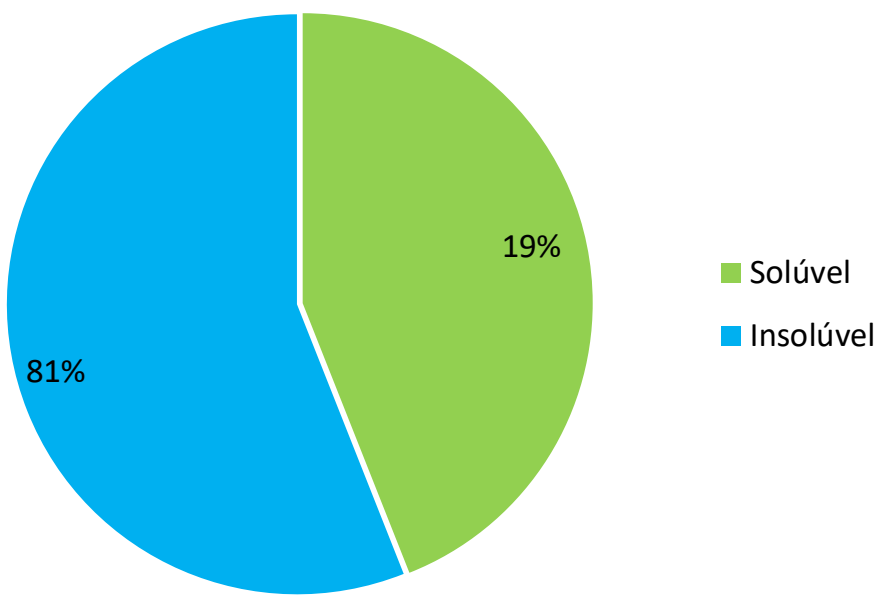

Figura 2. Porcentagem de material solúvel e insolúvel componente da PCl.

\subsubsection{Espectrometria de emissão óptica com plasma acoplado indutivamente (ICP-OES)}

A alíquota de $10 \mathrm{ml}$ do licor lixiviado foi devidamente diluído e após análise em ICPOES a concentração dos metais presentes na $\mathrm{PCl}$ foi determinada como pode ser visto na Tabela 1.

O cobre é o metal em maior concentração na $\mathrm{PCl}$, representando mais de $13,83 \%$ de sua composição, seguido do ferro com mais de $1,4 \%$ e do alumínio com $1,43 \%$.

Considerando somente os metais, a quantidade em massa de cobre é superior a soma de todos os outro metais. Isso ocorre devido a alta condutividade do cobre e sua aplicação nas camadas de trilhas da $\mathrm{PCI}(12)$.

Tabela 1. Resultados da análise de metais presente no licor lixiviado da água régia

\begin{tabular}{lcc}
\hline Metais & $\begin{array}{c}\text { \% em } \\
\text { massa } \\
\text { PCl }\end{array}$ & $\begin{array}{c}\text { \% em } \\
\text { massa } \\
\text { Metais }\end{array}$ \\
\hline Prata & 0,053 & 0,28 \\
\hline Alumínio & 1,43 & 7,56 \\
\hline Ouro & 0,069 & 0,36 \\
\hline Cobre & 13,83 & 73,10 \\
\hline Ferro & 1,47 & 7,77 \\
\hline Níquel & 0,32 & 1,69 \\
\hline Estanho & 1,7 & 8,99 \\
\hline Zinco & 0,043 & 0,23 \\
\hline Total & 18,92 & 100,0 \\
\hline
\end{tabular}

Apesar dos metais nobres representarem menos de $1 \%$ da $\mathrm{PCl}$, o valor intrínseco desses metais podem chegar a $80 \%$ do valor da placa (13). 


\subsubsection{Perda ao fogo}

O sólido não lixiviado na digestão em água régia, após filtrado e secado passou por ensaio de perda ao fogo. Após ser retirado do forno e pesado o resíduo apresentou perda de massa de $40,3 \%$. Esse valor representa o percentual em massa do sólido não lixiviado volatizado, ou seja, material polimérico. A quantidade de material não volatizado (cerâmico) presente na navícula foi de 59,7\%. Considerando que na digestão em água régia foi identificado que $18,9 \%$ da amostra inicial foi solubilizada, esta parcela corresponde a fração metálica da amostra inicial. Aplicando os percentuais das frações polímeras e cerâmicas identificadas no ensaio de perda ao fogo pode-se afirmar que a $\mathrm{PCl}$ estudada é composta ainda de $32,7 \%$ de polímero e $48,4 \%$ de cerâmicos, conforme Figura 3. Esses valores correspondem, em parte, ao verificado por Menetti (12).

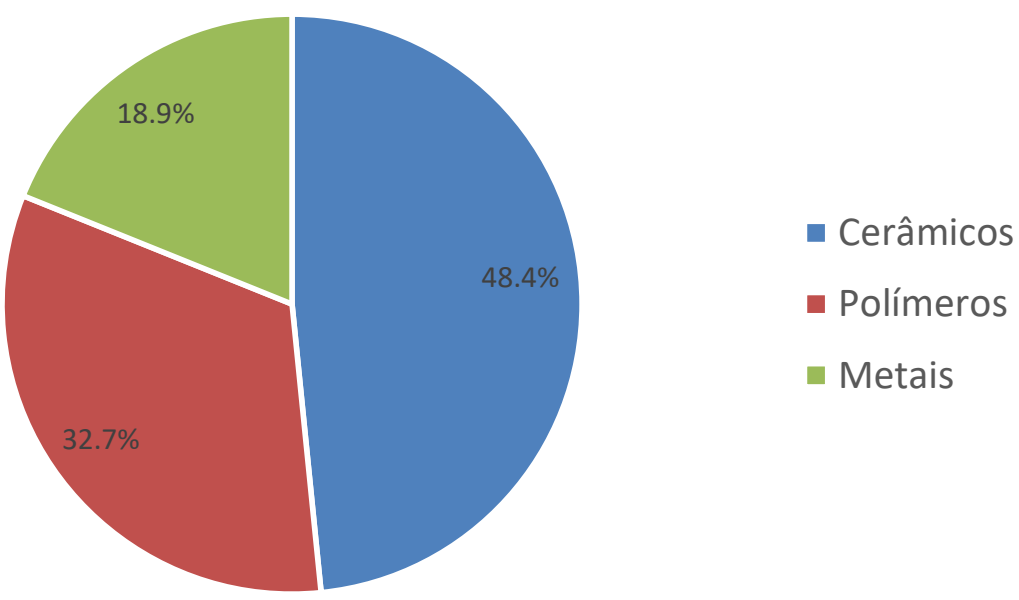

Figura 3. Composição dos materiais da PCl.

Com a identificação de prata nas PCls estima-se que a rota para a reciclagem de nanopartícula de prata pode ser através de moagem, separação magnética, separação eletrostática, extração sólido-liquido, extração liquido-liquido, cristalização e redução.

Sendo que a sintese de nanopartículas de prata pode seguir a rota de redução do nitrato de prata com a adição de citrato de sódio em $39,3 \mathrm{mM}$ e temperatura de $100^{\circ} \mathrm{C}$ com agitação método Turkevitch (14), através da obtenção de extrato lixiviado e purificado contendo nitrato de prata.

As nanopartícula de prata podem ser aplicadas como aditivo para materiais poliméricos, materiais adsorventes de gases ou metais e ainda em aplicações biotecnológicas.

\section{CONCLUSÃO}

Com o desenvolvimento do trabalho foi possível concluir:

- Do processo de comuinuição somente $1,46 \%$ de perda de materiais. 
- As PCls lead free são compostas de aproximadamente $19 \%$ de metais, $33 \%$ de polímeros e $48 \%$ de cerâmicas.

- A porcentagem dos metais presentes nas PCls correspondem a: 0,053\% de Ag; $1,43 \%$ de Al, $0,069 \%$ de $\mathrm{Au}, 13,83 \%$ de $\mathrm{Cu}, 1,47 \%$ de $\mathrm{Fe}, 0,32 \%$ de $\mathrm{Ni}, 1,7 \%$ de $\mathrm{Sn}$ e $0,043 \%$ de $\mathrm{Zn}$.

- 0,053\% de prata pode ser recuperada na forma de nanopartículas que podem ter aplicações em aditivo para materiais poliméricos, materiais adsorventes de gases ou metais e ainda em aplicações biotecnológicas.

\section{Agradecimentos}

Instituto Federal do Espírito Santo - IFES Campus Serra.

Departamento de Engenharia Química - Escola Politécnica - Universidade de São Paulo.

\section{REFERÊNCIAS}

1. Ilyas S, Ruan C, Bhatti HN, Ghauri MA, Anwar MA. Column bioleaching of metals from electronic scrap. Hydrometallurgy. 2010;101(3-4):135-40.

2. Oh CJ, Lee SO, Yang HS, Ha TJ, Kim MJ. Selective leaching of valuable metals from waste printed circuit boards. J Air Waste Manag Assoc. 2003;53(7):897-902.

3. Cayumil R, Khanna R, Rajarao R, Mukherjee PS, Sahajwalla V. Concentration of precious metals during their recovery from electronic waste. Waste Manag. 2016;57:121-30.

4. Havlik T, Orac D, Petranikova M, Miskufova A. Hydrometallurgical treatment of used printed circuit boards after thermal treatment. Waste Manag [Internet]. 2011;31(7):1542-6.

5. Official Journal of the European Union [página da internet] Directive 2002/96/EC of the European Parliament and of the Council on waste electrical and electronic equipment (WEEE). 2003 [acesso em 12 abr. 2017]. Disponível em: http://eur-lex.europa.eu/legal-content/EN/TXT/?uri=celex\%3A32012L0019

6. Official Journal of the European Union [página da internet]. Directive 2002/95/EC of the European Parliament and of the Council of 27 January 2003 on the restriction of the use of certain hazardous substances in electrical and electronic equipment (RoHS). 2003 [acesso em 12 abr. 2017]. Disponível em: http://eur-lex.europa.eu/legal-content/EN/TXT/?uri=CELEX:32002L0095.

7. Tuncuk A, Stazi V, Akcil A, Yazici EY, Deveci H. Aqueous metal recovery techniques from e-scrap: Hydrometallurgy in recycling. Miner Eng. 2012;25(1):28-37.

8. Kumari A, Jha MK, Lee JC, Singh RP. Clean process for recovery of metals and recycling of acid from the leach liquor of PCBs. J Clean Prod. 2016;112:4826-34.

9. Robinson $\mathrm{BH}$. E-waste: An assessment of global production and environmental impacts. Sci Total Environ. 2009;408(2):183-91.

10. Cui J, Zhang L. Metallurgical recovery of metals from electronic waste: A review. J Hazard Mater. 2008;158(2-3):228-56.

11. Jing-ying L, Xiu-li X, Wen-quan L. Thiourea leaching gold and silver from the 
printed circuit boards of waste mobile phones. Waste Manag.

2012;32(6):1209-12.

12. Menetti, RP; Chaves, AP; Tenório, JAS. Obtenção de Concentrados Metálicos Nãoferrosos a partir de Sucata Eletrônica. In: Anais do $51^{\circ}$ Congresso Anual da ABM. Associação Brasileira de Metalurgia e Materiais. Porto Alegre, RS. 1996.

13. Park YJ, Fray DJ, Recovery of high purity precious metals from printed circuit boards, Journal of Hazardous Materials. 2009; 164 1152-1158.

14. Turkevitch J; Stevenson, PC; Hilier J; Discuss. Faraday Soc. 1951, 11, 55. 